\title{
Failure to transfer or train a numerical discrimination using sequential visual stimuli in rats
}

\author{
HANK DAVIS and MELODY ALBERT \\ University of Guelph, Guelph, Ontario, Canada
}

\begin{abstract}
We previously reported that rats were able to discriminate among two, three, and four sequentially presented auditory stimuli (Davis \& Albert, 1986). In the present paper, we describe our failure to transfer this numerical discrimination to visual stimuli and to establish the same discrimination using visual stimuli in naive animals. These negative results conflict with sporadic evidence of intermodal (auditory-visual) transfer of numerical discriminations, although all such reports have involved simpler dichotomous (many vs. few) requirements. We believe the relative difficulty of our task may have confined processing to the single salient modality (audition) in which it was taught, and precluded evidence of abstract learning by rendering intermodal transfer unattainable.
\end{abstract}

It is generally believed that abstract learning has occurred when a discrimination is learned using stimuli of one modality and is transferred successfully to another (Meck \& Church, 1982; Roberts, 1982). Indeed, intermodal transfer of learning has been identified as an essential part of the definition of both number (Thompson, Mayers, Robertson, \& Patterson, 1970) and counting (Thorpe, 1956).

Despite the heuristic value of intermodal transfer, it is important to recognize that both species characteristics and task requirements may constrain how readily stimuli of different modalities may be interchanged on a learning task. For example, there is a growing body of evidence suggesting that not all sensory modes are equally suited to teaching a particular discrimination. Evidence of such sensory restrictions includes reports by Cohen, Escott, and Ricciardi (1984); Dawson (1961); Harsh, Badia, and Ryan (1984); and Wallace, Steinert, Scobie, and Spear (1980).

We recently reported a somewhat complex auditory discrimination, in which rats were taught to respond in the presence of three sequentially presented sounds, but not in the presence of either two or four sounds (Davis \& Albert, 1986). These results complement an earlier report by Fernandes and Church (1982) in which rats were taught to respond differentially in the presence of two and four sequential auditory signals. We argued that our results extended Fernandes and Church's findings by requiring an intermediate numerical discrimination (three vs. two or four), rather than a simpler dichotomous (many vs. few: four vs. two) discrimination.

\footnotetext{
This research was supported in part by Grant A0673 to the first author from the Natural Sciences and Engineering Research Council of Canada. The authors thank Rachelle Perusse for her critical comments. Address correspondence to Hank Davis, Department of Psychology, University of Guelph, Guelph, Ontario N1G 2W1, Canada.
}

Following the completion of this previous experiment, we investigated whether the discrimination, originally trained with sound, could be transferred to the visual mode. Our procedure involved retraining rat subjects in an operant chamber and exposing them to sequences of two, three, or four light stimuli. In addition, we attempted to train 3 naive animals, each of which had been shaped to leverpress but had received no prior discrimination training with light or sound.

The durations of visual stimuli and interstimulus intervals were identical to those successfully employed in our earlier experiment (stimulus length $=0.2$ or $0.5 \mathrm{sec}$; interstimulus intervals $=0.5$ or $0.9 \mathrm{sec}$ ). As in our previous experiment, and in that of Fernandes and Church (1982), stimulus length and total sequence durations were varied to ensure that successful performance was based upon numerical, and not durational, properties of the stimuli. Numerical cues were delivered by illuminating the stimulus light located over the lever on the front chamber panel. Later in training, as it became obvious that differentiation was not occurring, the overhead chamber light was dimly illuminated throughout the session in order to retain the signal property of the cue light, while minimizing any possible aversion associated with sudden change in illumination levels. The durations of light stimuli were programmed by using tapes of the original auditory stimuli to trigger occurrence of the light cues. In short, not only the stimulus values, but also their distribution over sessions, were identical to those of auditory stimuli previously used with success to train this discrimination.

One hundred twenty sessions were run for the transfer animals, and 70 sessions for new subjects. As in our previous experiment, all sessions consisted of 301 -min cycles, during which a particular numerical cue (two, three, or four) was repeatedly presented and response rate was recorded. Performance was assessed by comparing the 
response rate in the presence of three lights $(\mathrm{S}+)$ with that recorded during both two and four lights (S-).

At the end of original training with sound, response rates in the presence of $S+$ were approximately three times greater than those recorded for S- (see Davis \& Albert, 1986). This effect was eliminated altogether in the first animal, whose responding fell to virtual equivalence for all three stimuli during the initial transfer session. Although the second animal maintained a threefold rate difference during the first transfer session, this result was eliminated by the second session. The third animal maintained an approximately twofold rate difference between S+ and stimulus value "two" for approximately three sessions, before losing any consistent sign of differentiation. Although all animals showed sporadic evidence of an ability to discriminate between two and three stimuli, performance on the overall two-three-four discrimination never reached significance and never regained the levels originally recorded with auditory stimuli.

None of the 3 naive animals showed any sign of learning to discriminate among stimuli. There were no changes in response allocation over 70 test sessions, and response rates remained at virtual equivalence in the presence of the three numerical cues.

We have thus failed to transfer a previously established auditory discrimination to visual stimuli and failed to establish this discrimination using visual stimuli with naive animals. These results suggest that visual stimuli, at least the variety we have employed, are not suitable for training or transferring the present sequential numerical discrimination. However, our lack of success with visual stimuli cannot be generalized to all forms of numerical tasks. In fact, an attempt to transfer the auditory discrimination originally reported by Fernandes and Church (1982) to the visual mode has met with some degree of success. Church and Meck (1984) reported that rats "continue[d] to make a 'many' response following a sequence of 4-lights and a 'few' response to a sequence of 2-lights, although not to the extent that they did for sounds" (1984, p. 448). There is additional evidence of auditory-visual transfer of numerical discriminations, but each of these examples, like that of Church and Meck, involves a dichotomous task (e.g., Dawson, 1961; Lögler, 1959).

What accounts for our lack of success using sequential visual stimuli in the face of provisional evidence by Church and Meck (1984) and others? We believe that our failure to transfer or train successfully to a visual stimulus stems from the relative difficulty of our task (cf. Davis \& Albert, 1986; Fernandes \& Church, 1982). Although the procedures of Davis and Albert and of Fernandes and Church both involved the use of sequential stimuli, the latter procedure entailed training a considerably simpler many versus few dichotomy. In contrast, the Davis and Albert procedure required discrimination of an intermediate number from two adjacent values. Indeed, Dawson (1961) described this procedure as "an insoluble problem."
Although our animals were successful, this difference in difficulty may have resulted in a corresponding difference in the manner in which stimuli were processed.

This position is supported by evidence from species other than rats. Kuroda (1931) successfully trained a chimpanzee to discriminate between two and three visual stimuli, as well as between three and four. However, combining the tasks into a two-three-four discrimination resulted in a breakdown of performance to such an extent that the two versus three discrimination could not be recovered. Similarly, Dawson (1961) trained a jackdaw to discriminate on separate occasions between 2 and 3 stimuli and between 3 and 4 , but was unable to combine the tasks into a two-three-four discrimination.

In short, learning to differentiate many from few may be fundamental enough to occur at an abstract level, thus revealing itself in some degree of intermodal transfer (as Meck \& Church [1982] and others have reported). In contrast, the putatively more difficult numerical discrimination that we employed may have been processed strictly in terms of the auditory inputs with which it was originally trained, thus precluding successful transfer to a new modality.

If this view is correct, it also explains why new animals were incapable of learning the present numerical discrimination using visual cues. We believe that although sequential visual stimuli may support a relative numerousness (many versus few) judgment, such stimuli may be ill suited to the more complex level of processing necessary for success under a three adjacent number discrimination.

Our data underscore an interesting dilemma. On one hand, as Meck and Church (1982) and Thorpe (1956) argued, intermodal transfer may be a reasonable first step, perhaps even a criterion, for demonstrating a specialized sense of number in animals. Yet it may be the case, as our data suggest, that only the less complex forms of numerical competence (e.g., relative numerousness judgments) are learned in a manner abstract enough to support intermodal transfer. What then is the status of more complex numerical discriminations whose demonstration depends upon the use of stimuli from a particularly salient modality, and whose effects cannot be transferred to other stimuli?

\section{REFERENCES}

ChURCH, R. M., \& Meck, W. H. (1984). The numerical attribute of stimuli. In H. L. Roitblat, T. G. Bever, \& H. S. Terrace (Eds.), Animal cognition. Hillsdale, NJ: Erlbaum.

CoHEn, J. S., EscotT, M., \& Ricciardi, P. (1984). The role of reinforcement symmetry and stimulus modality in successive delayed matching to sample in the rat. Canadian Journal of Psychology, 38, 63-79.

DAvIs, H., \& AlBerT, M. (1986). Numerical discrimination by rats using sequential auditory stimuli. Animal Learning \& Behavior, 14, 57-59.

Dawson, E. (1961). Counting in jackdaws. Behavior, 18, 229-238. Fernandes, D. M., \& ChURCH, R. M. (1982). Discrimination of the 
number of sequential events by rats. Animal Learning \& Behavior, 10, 171-176.

HARSh, J., BADIA, P., \& RyAN, K. (1984). Signal modality and choice between signaled and unsignaled food. Journal of the Experimental Analysis of Behavior, 42, 279-289.

KURODA, R.(1931). On the counting ability of a monkey. Journal of Comparative Psychology, 12, 171-180.

LögleR, P. (1959). Versuche zur Frage des 'Zähl'-Vermögens an einen Graupapagei und Vergleichsversuche an Menschen. Zeitschrift für Tierpsychologie, 16, 179-217.

MeCK, W. H., \& CHURCH, R. M. (1982). Abstraction of temporal attributes. Journal of Experimental Psychology: Animal Behavior Processes, 8, 226-243.
RoBerTs, S. (1982). Cross-modal use of an internal clock. Journal of Experimental Psychology: Animal Behavior Processes, 8, 2-22.

Thompson, R. F., Mayers, K., Robertson, R., \& Patterson, C. (1970). Number coding in association cortex of the cat. Science, 168 , 271-273.

THORPE, W. H. (1956). Learning and Instinct in Animals. London: Methuen.

Wallace, J., Steinert, P. A., Scobie, S. R., \& Spear, N. E. (1980). Stimulus modality and short-term memory in rats. Animal Learning \& Behavior, 8, 10-16.

(Manuscript received for publication March 9, 1987.) 